\title{
Allergische Alveolitis nach Grippeschutzimpfung
}

\author{
Allergic Alveolitis After Influenca Vaccination
}

Autoren

Institute
D. Heinrichs' ${ }^{1}$, J. Sennekamp ${ }^{2}$, A. Kirsten ${ }^{3}$, D. Kirsten ${ }^{1}$

Krankenhaus Großhansdorf, Zentrum für Pneumologie und Thoraxchirurgie

Allergologisch-Immunologisches Labor, Bonn

Pulmonary Research Institute, Großhansdorf eingereicht 3.6. 2009

akzeptiert nach Revision 17. 6.2009

Bibliografie

Dol $10.1055 / \mathrm{s}-0029-1214909$

Online-Publikation: 25. 8. 2009

Pneumologie 2009; 63:

508-511 @ Georg Thieme

Verlag KG Stuttgart · New York ISSN 0934-8387

Korrespondenzadresse Dr. D. Heinrichs

Krankenhaus Großhansdorf Zentrum für Pneumologie und Thoraxchirurgie

Wöhrendamm 80

22927 Großhansdorf

d.heinrichs@

kh-grosshansdorf.de

\section{Zusammenfassung \\ $\nabla$}

Allergische Alveolitiden als Nebenwirkungen von Impfungen sind sehr selten. Wir berichten über eine lebensbedrohliche Komplikation bei einer Patientin nach Influenza-Impfung. Das ursächliche Antigen für die allergische Reaktion war das Grippevirus selbst. Unsere Patientin litt an einer exogen-allergischen Alveolitis seit 12 Jahren. Aufgrund der aktuellen Empfehlungen zur jährlichen Grippeschutzimpfung bei Patienten mit chronischen Lungenerkrankungen sind weitere Untersuchungen von Patienten mit vorbestehender Diagnose einer exogen-allergischen Alveolitis von großer Bedeutung für die Arzneimittelsicherheit.

\section{Einleitung \\ $\nabla$}

Allergische Alveolitiden durch Impfungen (oder Impfstoffe) sind noch nicht lange bekannt und große Raritäten. Erste Fallberichte über eine allergische Alveolitis nach intravesikaler BCG (Bacille Calmette-Guerin)-Applikationen erfolgten 1987 und $1992[1,2]$. Später folgten je eine Beschreibung einer Alveolitis durch Impfung mit Hepatitis-B-Impfstoff [3], Masern-Impfstoff [4] und Anthrax-Impfstoff [5].

Eine exogen-allergische Alveolitis (EAA) nach Influenza-Impfung wurde bisher erst ein einziges Mal beschrieben [6]. Wir präsentieren hier einen zweiten, weitaus schwereren Fall, bei dem erstmalig gezeigt wird, dass diese Immunreaktion mit einem IgG-Antikörper im Serum gegen das Grippevirus selbst assoziiert ist.

\section{Fallbericht}

$\nabla$

Bei einer 59-jährigen Patientin wurde vor 12 Jahren eine exogen-allergische Alveolitis auf Sittiche (Vogelhalterlunge) diagnostiziert.

\section{Abstract \\ $\nabla$}

Allergic alveolitis as a side effect of vaccination is very rare. We report a life-threatening complication in a female patient after influenza vaccination. The causative antigen was the influenza virus itself. Our Patient has suffered from exogen-allergic alveolitis for 12 years. Because of the guidelines of regular administration of influenza vaccination in patients with chronic pulmonary disease further research in patients with known exogen-allergic alveolitis is vitally important for the pharmaceutical drug safety.

Trotz weitgehender Allergenkarenz und wechselnder Kortisonmedikationen kam es klinisch, lungenfunktionell und auch computertomografisch zu einem langsamen Progress der Erkrankung im Sinne einer chronischen EAA.

Begleitende serologische Untersuchungen vor Evaluation zur Lungentransplantation ergaben eine chronische Hepatitis-B-Infektion mit niedriger Viruslast. Die Leberwerte waren unauffällig. Die Lebersyntheseleistung der Patientin war komplett erhalten. Medikamentös bestand eine Prednisolon-Dauertherapie mit $10 \mathrm{mg} / \mathrm{d}$.

Eine Sauerstofflangzeit-Therapie (LTOT) wurde seit 2007 durchgeführt. Entsprechend den Empfehlungen der DGP erfolgten jährliche Grippeschutzimpfungen. Nur 2007 hatte die Patientin auf die Impfung verzichtet, da sie im Jahr 2006 vorübergehend über Arthralgien und Unwohlsein nach der Impfung gelitten hatte.

Sechs Tage nach der diesjährigen Grippeschutzimpfung mit Begrivac ${ }^{\circledR}$ traten zunächst Gelenkschmerzen, dann Atemnot und Fieber um $38^{\circ} \mathrm{C}$ auf. Wegen zunehmender klinischer Verschlechterung erfolgte nach zehn Tagen die stationäre Aufnahme. Die Patientin war inzwischen zyanotisch und ikterisch. Beidseits über den Lungen 

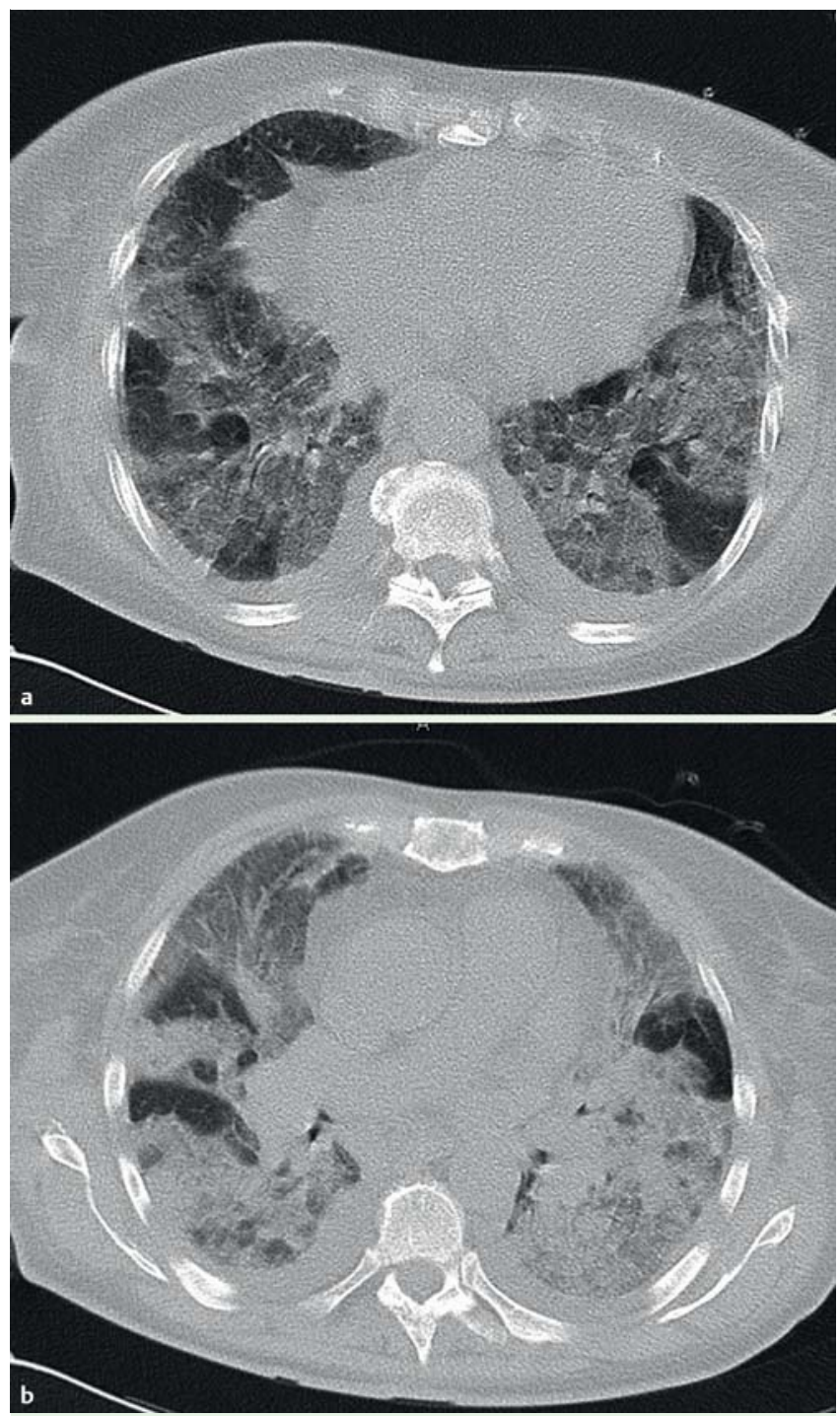

Abb. 1 a Das computertomografische Bild zeigt ausgedehnte milchglasartige Infiltrate mit sowohl zentraler als auch peripherer Verteilung unter Freilassung einzelner landkartenartiger regelrecht transparenter Lungenareale. b Alveoläre Infiltrate fanden sich betont in den Lungenober- und unterfeldern.

war inspiratorisch Knistern auskultierbar. Die Leukozyten waren mit 12,6/nl erhöht. Das CRP stieg auf maximal 232,5 mg/l. Weiter pathologisch erhöht waren GOT mit 51 U/l, GPT mit 90 U/1, Gamma-GT mit 417U/l, AP mit 472 U/l, Bilirubin mit 5,7 mg/dl und LDH mit 520U/1. Als Marker der Herzinsuffizienz erfasste man eine vorübergehende Erhöhung des ProBNP auf maximal $10606 \mathrm{pg} / \mathrm{ml}$ (normal bis $220 \mathrm{pg} / \mathrm{ml}$ ).

Es bestand eine schwere respiratorische Partialinsuffizienz mit einem $\mathrm{pO}_{2}$ von $45 \mathrm{mmHg}$ trotz Gabe von $8 \mathrm{l} \mathrm{O}_{2} /$ min über eine $\mathrm{Na}$ sensonde.

Das computertomografische Bild war gekennzeichnet durch ausgedehnte milchglasartige Infiltrate mit sowohl zentraler als auch peripherer Verteilung unter Freilassung einzelner landkartenartiger regelrecht transparenter Lungenareale ( $\bullet$ Abb. 1 a). Alveoläre Infiltrate fanden sich betont in den Lungenober- und unterfeldern ( $\bullet$ Abb.1 b).

CT-Thorax und Echokardiographie wiesen auf eine pulmonale Hypertonie hin. Im Rahmen der Rechtsherzdekompensation fanden sich beidseits geringe Pleuraergüsse. Die linksseitigen Herz- höhlen waren normal groß, sodass ein kardiales Ödem ausgeschlossen schien. Auf eine bronchoalveoläre Lavage wurde aufgrund der Schwere des Krankheitsbildes verzichtet.

Die Patientin musste über ca. eine Woche nicht-invasiv beatmet werden. Zusätzlich verordneten wir hochdosiert Prednisolon $100 \mathrm{mg} / \mathrm{d}$, eine Antibiotikatherapie mit Tazobactam ${ }^{\circledR}(3 \times 4,5 \mathrm{~g}$ pro Tag) sowie niedrig dosiert Diuretika. Hierunter kam es sowohl klinisch als auch computertomografisch innerhalb von 2 Wochen zu einer Besserung. Die Milchglastrübungen waren deutlich rückläufig, die Pleuraergüsse nicht mehr nachweisbar. Die Laborparameter waren kontinuierlich rückläufig und normalisierten sich mit Ausnahme der alkalischen Phosphatase (105 U/l) und Gamma-GT (110 U/1). Die LTOT konnte auf den prästationären Bedarf von 3 1/min reduziert werden. Die Entlassung erfolgte mit einer Prednisolondosis von zunächst 20 mg/d.

\section{Techniken der Antikörpernachweise}

\section{$\nabla$}

Die IgG-Antikörper der Patientin und der Kontrollpersonen wurden in üblicher Weise mittels Festphasen-ELISA nachgewiesen [7]. Der Grippeimpfstoff Begrivac ${ }^{\circledR}$ wurde 1:1000 mit CoatingPuffer (0,1 molarer Na-Carbonat-Puffer pH 9,6) verdünnt. Je eine Probe dieser Verdünnung wurde in MaxiSorp ${ }^{\mathrm{TM}}$-Näpfchen in Mikrotiterplatten (Fa. Thermo Fisher Scientific, Schwerte) gefüllt, damit sich der Grippeimpfstoff bei einer Inkubationszeit von 24 Stunden an die Wände dieser Gefäße bindet (coating). Überschüssiges Antigen wurde anschließend mit PBS dreimal ausgewaschen. Die Antigen beschichteten Näpfchen wurden im üblichen IgG-Festphasen-ELISA mit Peroxidase im Allercoat-System ${ }^{\circledR}$ bei $37^{\circ} \mathrm{C}$ verwendet. Als Kontrollen wurden jeweils 12 Seren von Grippe geimpften und nicht Grippe geimpften Personen verwendet.

Die IgG-Antikörper gegen Nymphensittich- und Wellensittichserum wurden mit derselben Technik nachgewiesen.

Die IgE- und IgG-Antikörper gegen Hühnerei-Eiweiß wurden mittels CAP FEIA der Fa. Phadia gemessen.

\section{Ergebnisse der Antikörperuntersuchungen}

Bei der ersten Untersuchung im Jahre 1996 wurden bei einer klinisch manifesten Vogelhalterlunge mit Sittichen stark positive IgG-Antikörper-Spiegel sowohl gegen Nymphensittichserum als auch gegen Wellensittichserum nachgewiesen. Eine Kontrolluntersuchung im Jahr 2000, nach inkonsequenter Allergenkarenz (die Patientin gab Kontakt zu anderen Sittichhaltern an), ergab mittelstark positive IgG-Antikörper gegen Nymphensittich und schwach positive IgG-Antikörper gegen Wellensittich sowie gegen die Schimmelpilze Aspergillus fumigatus und Aspergillus terreus, Aureobasidium pullulans, Cephalosporium acremonium und Cryptostroma corticale.

Jetzt, nach weitgehender Vogelkarenz, waren nur noch fraglich positive Antikörper gegen Nymphensittichserum nachweisbar. Gegen Huhnserum und Hühner-Ei waren weder IgG- noch IgEAntikörper nachweisbar, sodass keine Hühnereiweiß-Allergie vorlag.

Im IgG-ELISA auf den Grippeimpfstoff (Begrivac ${ }^{\circledR}$ ) reagierte das Serum der Patientin eindeutig stark positiv im Vergleich zu den Kontrollen ( $\bullet$ Abb. 2). 


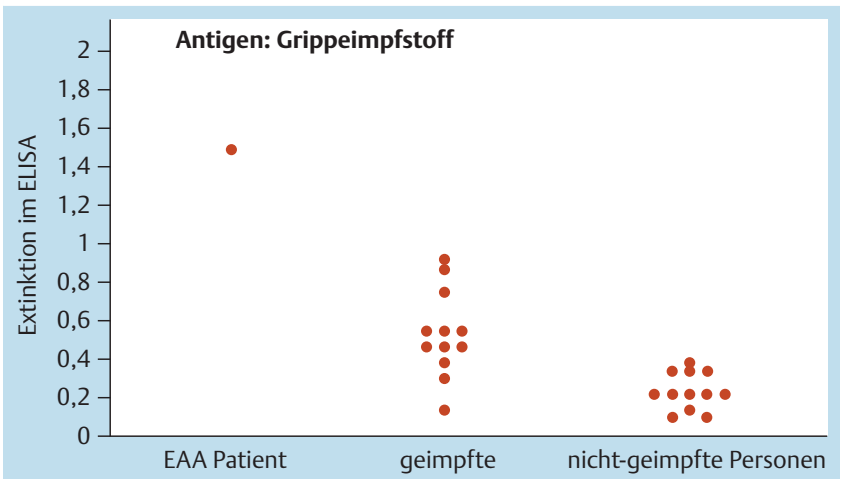

Abb. 2 IgG-Antikörper gegen Influenza-Impfstoff der Patientin, geimpften und nicht-geimpften Kontrollpersonen.

\section{Diskussion \\ $\nabla$}

Die Diagnosekriterien einer exogen-allergischen Alveolitis sind bei der Patientin bei einem eindeutigen Zusammenhang mit einem kausalen Antigen, der charakteristischen klinischen Symptomatik, mit Rasselgeräuschen, den typischen Laborbefunden einer Leukozytose und einem erhöhten CRP, einem typischen Röntgenbefund mit Milchglastrübung (s. $\odot$ Abb. 1) und einer schweren Diffusionsstörung sowie dem Nachweis stark positiver IgGAntikörper eindeutig erfüllt [8].

Die respiratorische Insuffizienz war bei unserer Patientin noch ausgeprägter als bei der Erstbeschreibung [6], sodass in beiden Fällen eine hochdosierte Sauerstoffgabe, in unserem Fall sogar die nicht-invasive Beatmung, erforderlich wurde.

Die Ursache für die Pleuraergüsse, den Anstieg der Leberparameter und des ProBNP ist im Rahmen der akuten massiven Rechtsherzdekompensation zu sehen.

Bei der Erstbeschreibung einer EAA nach Grippeschutzimpfung traten die Symptome bei dem Patienten erst 10 Tage nach der Impfung auf [6] und nicht wie bei unserer Patientin bereits nach 6 Tagen. Auch bei einer allergischen Pneumonitis auf eine Hepatitis-B-Impfung trat die pulmonale Reaktion erst zwei Tage nach der Impfung auf [3]. Diese langen Latenzzeiten zwischen Antigenkontakt und dem Beginn der Symptome sind bei der exogenallergischen Alveolitis durch inhalative Antigene nicht bekannt. Hier beträgt die Latenzzeit in der Regel 4-6 Stunden bis maximal 12 Stunden [9]. Von medikamentös ausgelösten allergischen Alveolitiden wie bei der Amiodaron-Lunge ist der Zeitpunkt der Sensibilisierung nie bekannt. Erst die einsetzende Atemnot und die Kenntnis eines möglichen derartigen Zusammenhangs lenkt den Verdacht auf eine Immunreaktion durch Medikamente. Bei der bekannten chronischen Vogelhalterlunge der Patientin war das Immunsystem der Lunge zum Zeitpunkt der Impfung hochreguliert und damit wahrscheinlich empfänglich für eine neuerliche Sensibilisierung nach mehrfachen Grippeimpfungen gegen das Grippevirus. Dass eine allergische Alveolitis die Immunzellen der Lunge langfristig verändert, dafür spricht die meist zu beobachtende persistierende Lymphozytose in der bronchoalveolären Lavage. Selbst unter Allergenkarenz bleibt die Lymphozytose noch über viele Jahre bestehen und schwächt sich nur langsam ab [10], abhängig von der Expositionsprophylaxe.

Selbst asymptomatische Personen mit IgG-Antikörpern gegen EAA-Antigene weisen oft eine, wenn auch weniger stark ausgeprägte BAL-Lymphozytose auf $[9,10]$. Interessanterweise wies der Patient der bislang einzigen Beobachtung einer Grippe- schutzimpfung induzierten Alveolitis einen IgG-Antikörper gegen Taubenprotein auf, ohne dass bei ihm vor der Impfung EAASymptome existiert hätten. Somit ist auch bei ihm von einem hochregulierten pulmonalen Immunsystem auszugehen, das auf die Impfung in der genannten Weise reagieren kann.

Aus der Literatur ist auch das Auftreten einer Autoimmunerkrankung nach Grippeimpfung wie der ANCA assoziierten mikroskopischen Polyangiitis bekannt. Die Immunreaktion erfolgte erst 7 Tage nach der Impfung [11], ein weiterer Beleg für die benötigte Zeit bei hämatogener Sensibilisierung durch den Grippeimpfstoff.

Bei einer Latenzzeit von über einer Woche wird vom diagnostizierenden Arzt sicherlich selten ein Zusammenhang zwischen der Impfung und den pulmonalen Krankheitssymptomen vermutet. Man darf deshalb spekulieren, dass eine beachtenswerte Dunkelziffer bei den von Impfungen hervorgerufenen allergischen Alveolitiden existiert. In Kenntnis dieses Zusammenhangs scheint es wichtig, auf die Möglichkeit derartiger unerwünschter Immunreaktionen von Impfstoffen aufmerksam zu machen. Die EAA-Arbeitsgemeinschaft wird sich im nächsten Jahr dieses Themas annehmen.

Das ursächliche Antigen für die allergische Reaktion unserer Patientin auf den Grippeimpfstoff war das Grippevirus selbst, da IgE- und IgG-Antikörper gegen das Hühnereiweiß in dem Grippeimpfstoff nicht nachweisbar waren. Die IgG-Antikörper gegen das verwandte Nymphensittichserum waren zur Zeit der allergischen Reaktion nur noch schwach nachweisbar.

Bei der Erstbeschreibung durch Johnston waren keine Untersuchungen auf Influenza-Antikörper erfolgt. Somit bleibt unklar, ob sich die Immunreaktion der ersten Fallbeschreibung gegen Hühnereiweiß oder gegen das Influenzavirus gerichtet hatte.

Der Nachweis von IgG-Antikörpern gegen Vogelproteine in beiden Fallbeschreibungen scheint ein zusätzlicher Risikofaktor für das Auftreten von Alveolitiden nach Grippeschutzimpfungen zu sein.

Die Erstbeschreiber der Grippeimpfstoff-Alveolitis berichten, dass den britischen Gesundheitsbehörden zwei weitere, nicht publizierte Fälle bekannt seien [6]. Das deutsche Paul Ehrlich Institut teilte uns nach unserer Nebenwirkungsmeldung mit, dass dem Institut bisher in Deutschland kein einziger gemeldeter Fall einer interstitiellen Pneumonie nach Grippeimpfung bekannt sei. Weitere Untersuchungen von Patienten mit Vogelhalterlunge auf die Verträglichkeit der Grippeimpfung sind nach der Kenntnis dieser beiden mitgeteilten lebensbedrohlichen Lungenreaktionen von großer Bedeutung für die Arzneimittelsicherheit.

Interessanterweise reagierte unsere Patientin auf die vorhergehende Impfung bereits mit Gelenkbeschwerden. Da bei anderen Impfungen wie beispielsweise der Tetanus-Impfung erhöhte IgG-Antikörpertiter gegen den Impfstoff (hohe Impftiter) mit Unverträglichkeiten auf Impfungen assoziiert sind, ist zu vermuten, dass auch hier die Bestimmung des Impftiters vor einer Grippeimpfung einen Beitrag zur Arzneimittelsicherheit liefern könnte, d.h. bei hohem Impftiter müsste von der Grippeimpfung abgesehen werden.

Der hier vorgestellte Fall zeigt, dass derartige Impfreaktionen an der Lunge nach Grippeschutzimpfungen nicht nur durch eine Hühnereiweißallergie, sondern auch durch das Virus selbst induziert werden können. Es gilt diese Nebenwirkung zu kennen, um bei ähnlich gelagerten Fällen klinisch richtig zu entscheiden. Darüber hinaus sollte durch eine Studie bei grippeschutzgeimpften Patienten mit EAA das mögliche Risiko einer derartigen Reaktionsweise geklärt werden. 


\section{Interessenkonflikte}

$\nabla$

Keine angegeben.

\section{Literatur}

1 Israël-Biet $D$, Venet $A$, Sandron $D$ et al. Pulmonary complications of intravesical Bacille Calmette-Guérin immunotherapy. Am Rev Resp Dis 1987; 135: $763-765$

2 Molina JM, Rabian C, D'Agay MF et al. Hypersensitivity systemic reaction following intravesical bacillus Calmette-Guerin: successful treatment with steroids. J Urol 1992; 147: 695-697

3 Allen MB, Cockwell P, Page RL. Pulmonary and cutaneous vasculitis following hepatitis B vaccination. Thorax 1993; 48: 580-581

4 Measles pneumonitis following measles-mumps-rubella vaccination in a patient with HIV infection. MMWR 1996; 45: 603-606
5 Timmer SJ, Amundson DE, Malone JD. Hypersensitivity pneumonitis following Anthrax vaccination. Chest 2002; 122: 741 - 745

6 Johnston SD, Kempston A, Robinson TJ. Pneumonitis secondary to the influenza vaccine. Postgrad Med J 1998; 74: 541 - 542

7 Sennekamp J, Koschel D, aufm Kampe A et al. Serumdiagnostik der Zimmerspringbrunnen-Alveolitis. Atemw Lungenkrkh 2005; 31: 182 - 187

8 Arbeitsgemeinschaft exogen-allergische Alveolitis. Empfehlungen zur Diagnostik der exogen-allergischen Alveolitis. Pneumologie 2007; 61: $52-56$

9 Sennekamp J. Extrinsic allergic Alveolitis - hypersensitivity pneumonitis. München: Dustri Verlag, 2004

10 Costabel U. Berufsbedingte exogen-allergische Alveolitis. In: Konietzko N, Costabel U, Bauer PC. Lunge und Arbeitswelt. Berlin: Springer, 1990: $25-48$

11 Uji M, Matsushita $H$, Iwata S. Microscopic polyangiitis after influenza vaccination. Int Med 2005; 44: $892-896$ 